\begin{tabular}{|l|l|}
\hline Journal of & \\
Radiotherapy & $\begin{array}{l}\text { Journal of Radiotherapy in Practice (2018) } \\
17,137-141 \quad \text { O Cambridge University Press } 2018 \\
\text { in Practice }\end{array}$ \\
\hline
\end{tabular}

\title{
In this issue
}

I am pleased to introduce the 'in this issue' for the second issue of the Journal of Radiotherapy in Practice for Volume 17 published in June 2018. In this issue there are 11 original articles on a range of topics, a technical note on the imaging dose of megavoltage computed tomography (MVCT) for treatment verification in the tomotherapy of breast cancer and a case report on the radiation therapists' compliance to a palliative imaging protocol.

The first paper, written by Flinton, Singh and Haria, is on the readability of internet-based patient information for radiotherapy patients. Information is key to patient informed choice and the internet is currently a major source of health information for adults in the United Kingdom. In order for the users to make use of the information it must be presented in a way that the user can understand. This depends on a number of factors one being that the document is written at the right level to be understood by the reader, readability. The aim of this study was to assess the readability of radiotherapy-related documents on the internet and compare their levels to published norms.

An internet search was undertaken using Google, to identify UK-based literature. Once identified documents were downloaded into Word and cleaned of punctuation other than that at the end of the sentence, documents were then analysed by the software package Readability Studio.

The authors conclude that documents tended to be written at too high a reading level, but the reading level had improved from a similar study conducted in 2006. The level of readability appears to show a relationship to the use of passive voice, which was very variable in the sample collected and reduction in the use of passive voice could help with the readability of the information.
In the next paper, Chauhan, Holch and Holborn, present their paper on the assessment of the information and support needs of radical prostate cancer patients and acceptability of a groupbased treatment review. Current literature suggests the information and support needs of oncology patients undergoing radical radiotherapy to the prostate often remain unmet and can impact the quality of life. The authors explore the effectiveness of delivery and opportunities for service improvement, including a group-based treatment review.

In total, 60 prostate patients completing radical radiotherapy (mean age 70 , range 47-79) in a UK cancer centre completed a self-designed questionnaire assessing information and support. To explore views on a group-based treatment review, $11 \%$ took part in a semi-structured interview. Descriptive data were computed and interviews transcribed and analysed thematically.

The authors found overall, patients felt their needs were being met. Suggestions for improvement (more information on preparation, side effects and delays) will be implemented locally. Future work will explore the feasibility of group reviews in patients undergoing radical radiotherapy to the prostate.

In the next paper, Chow, Runqing and Markel undertake a study on the dosimetric variations in calculation grid size in prostate volumetricmodulated radiotherapy (VMAT): A dose-volume histogram (DVH) analysis using the Gaussian error function (GEF). Varying the calculation grid size can change the results of dose-volume and radiobiological parameters in a treatment plan and therefore have an impact on the treatment planning quality assurance. This study investigated the dosimetric influence of the calculation grid size variation in the prostate VMAT plan.

Dose distributions of ten prostate VMAT plans were acquired using calculation grid sizes of 
1-5 mm. DVH analysis was carried out to determine the dose-volume variation corresponding to the grid size change using the Gaussian error function. At the same time, dosevolume points, dose-volume parameters and radiobiological parameters were calculated based on DVHs of targets and organs at risks (OAR) for each grid size.

The authors conclude that, knowing the dosimetric variation in this study is important to the radiotherapy staff in the quality assurance for prostate VMAT planning.

In the next paper, Radaideh, undertakes an evaluation of the thermoplastic Klarity mask use during intensity-modulated radiation therapy (IMRT) on skin doses for head and neck carcinoma. This was a prospective study of five nasopharyngeal cancer patients submitted to IMRT. An anatomical perspex head and neck phantom was designed and used. All treatment plans of the five patients were transferred to the phantom, separately. All measurements were performed using chip-shaped thermoluminescent dosimeters (LiF:Mg, Ti TLDs). The TLDs were distributed on the targets at the phantom, by then irradiated and TLDs measurements were taken with and without the Klarity Mask. Three fractions for each patient plan were performed and compared with the treatment planning system doses as guided by computed tomography (CT).

The author concludes the treatment planning system may not give accurate dose values at the surface. Klarity mask used for patient immobilisation increase surface dose by $10.83 \%$ more than that without the mask, however, skin reaction and the surface dose measurements should be monitored, and must be taken into account.

In the paper by Atiq, Atiq, Iqbal, Shamsi and Buzdar, the aim of the research was to investigate tolerance dose to OARs as well as degree of conformity and homogeneity for head and neck cancer patients by using simultaneous integrated boost intensity-modulated radiotherapy technique (SIB IMRT).

This study analysed 15 head and neck cancer patients receiving treatment using inverse planned SIB IMRT technique. Using beam energy of $6 \mathrm{MV}$, two dose levels of 70 and 55.4 Gy were used to treat the tumour. Doses of $2 \mathrm{~Gy}$ in 35 fractions and $1.68 \mathrm{~Gy}$ in 33 fractions were simultaneously delivered for effective planning target volume (PTV1) and boost planning target volume (PTV2), respectively.

Authors found that dose distribution in PTV1 and critical organs lies within tolerance dose guidelines protecting spinal cord, brainstem, optic chiasm, optic nerve, thus reducing the risk of damage to normal tissues. Minor deviation from tolerance limit was observed for parotid glands. This technique provided highly conformal and homogenous dose distribution as well as better sparing of OARs, hence verifying quality assurance results to be satisfactory. The authors conclude, SIB IMRT technique offers best solution for preserving organ function by keeping dose below tolerance level. Treatment of head and neck carcinoma using SIB IMRT is feasible, more efficient and dose escalation is achieved in a single plan.

In the next paper, Siow and Lim, present a new approach to pre-planning using virtual simulation. For palliative radiotherapy treatments, two types of simulation are available at their Centre: conventional (or 2-D) and virtual. Each has its advantages: conventional simulation requires less preparation time whereas virtual simulation allows accurate visualisation and identification of target volumes in threedimension (3D). The authors propose a new approach where treatment field parameters are determined on diagnostic CT scans, and then reproduced with reference to a patient's bony landmarks using conventional simulation. This combines the benefits of both methods. They argue that the slight differences in set-up between diagnostic and simulation CT scans will have little impact on the determination of the target volume in palliative treatment settings.

In total, 12 patients who had diagnostic scans performed within a month before their virtual simulation were randomly selected. Both scans were retrieved retrospectively for the study. An independent radiation oncologist contoured the target volumes on both scans and their relative 
positions were compared by fusing the digitally reconstructed radiographs generated from the respective scans. A $2 \mathrm{D}$ Conformity Index was then calculated and tabulated for $0,0 \cdot 5,1 \cdot 0,1 \cdot 5$ and $2 \mathrm{~cm}$ margins to evaluate the accuracy of this approach and determine the margins required to account for the inherent variability of this method. In addition, the deviation or offset of the centre of field was also measured and analysed.

Authors conclude that this pilot study suggests that this approach is feasible. They recommend adding an additional margin of up to $0.5 \mathrm{~cm}$ (to the usual treatment margins) to ensure good coverage of the target volume when using this method.

In the next paper, authors Sakthivel, Mani, Mani and Boopathy undertake a study to calculate organ equivalent dose (OED) and to estimate excess absolute risks, lifetime attributable risks (LAR) and relative risks (RR) from stereotactic ablative radiotherapy (SABR) for lung cancer to infield, close to field and out of field structures.

A total of five patients with T1, T2 $(4 \mathrm{~cm}), \mathrm{N} 0$, M0 medically inoperable non-small cell lung cancer was selected for treatment planning. Patient selection criteria were based on RTOG 0236. Five treatment deliveries were investigated (i) 3D conformal radiotherapy, (ii) IMRT, (iii) intensity-modulated radiotherapy with flattening filter-free beam (IMRTF), (iv) VMAT, (v) VMAT with FFF arcs (VMATF). Delineated normal structures included chest wall, left and right lung, trachea, small and large airways, spinal cord, oesophagus and involved ribs. All plans were prescribed to $60 \mathrm{~Gy}$ in five fractions to primary PTV volume so that $\geq 98 \%$ of the PTV received $\geq 98 \%$ of the prescription dose and ITV received $100 \%$ of the prescription dose. The OED for all delineated normal structures was calculated using differential DVHs. Using risk models, the age-dependent LAR's and RR were calculated. In addition, the secondary cancer risk for organs inside primary radiation was analysed using sarcoma and carcinoma risk models.

Authors report, from a clinical perspective, it should be concluded that all five solutions investigated in the study can offer high quality of patient treatments and only estimates of radiation-induced malignancies can truly differentiate among them. The results suggested that it would be reasonable to use the cumulative LAR difference when we need to select between treatment techniques. In conclusion, the LAR of radiation-induced secondary cancer was significantly lower when using VMATF than when using IMRT for SABR lung patients. VMATF would be the right choice for the treatment of SABR lung patients in terms of LAR. However, more work is required for the specific estimation and long-term validation and updating of the models behind LAR estimation.

In the paper by Lee and Kim, authors undertake a study on practical lookup tables for ensuring target coverage in a clinical setup for skin cancer electron therapy. The aim of the study was to create practical lookup tables containing per cent depth dose (PDD) and profile parameters of electron beams and to demonstrate clinical application of the lookup tables to skin cancer treatment to ensure target coverage in a clinical setup.

The method used for 6 and $9 \mathrm{MeV}$ electron energies, PDDs and profiles at clinically relevant depths [i.e., R95 (distal depth of 95\% maximum dose), R90, R85 and R80] were measured in water at $100 \mathrm{~cm}$ source-to-surface distance for an $10 \times 10 \mathrm{~cm}^{2}$ open field and circular cut-outs with diameters of $4,5,6,7$ and $8 \mathrm{~cm}$. Then PDD parameters along with profile parameters such as width of isodose lines and penumbra at the clinically relevant depths were determined. Output factors for the cut-outs were measured at $\mathrm{d}_{\max }$ in water and solid water.

The authors conclude that dosimetry lookup tables for electron beam therapy should include profile parameters at clinically relevant depths and be provided to clinicians to ensure target coverage in a clinical setup.

In the next paper by Aghdam, Baghani and Aghdam, undertake a study to estimate the cancer risk incidence for different body organs due to accidentally released radionuclides from the Bushehr Nuclear Power Plant (BNPP). 
The assumed hypothesis was atmospheric dispersion of radionuclide into the environment due to the safety failure of BNPP. Total effective dose equivalent from radionuclide diffusion in the medium was calculated using HOTSPOT code at two different wind speeds. Finally, the risk of cancer incidence for different organs of male and female sex was estimated by BEIR VII model.

The authors report, based on their results, it can be concluded that younger persons are more subject to the cancer risk incidence because of both the intrinsically greater radio-sensitivity of their organs and their longer remaining life expectancy during which a cancer may develop. The overall risk of cancer incidence as well as the site-specific solid cancer incidence was highly dependent to the sex of exposed person, so that the female sex was more exposed to the cancer risk incidence at all of the irradiation levels understudy.

In the study by Chopra, Rai, Sethi, Avadhani and Kehwar, authors investigate discrepancies in dose calculation algorithms used for lung stereotactic body radiotherapy (SBRT) plans. In total, 30 patients lung SBRT treatment plans, initially generated using BraiLab Pencil Beam (BL_PB) algorithm for $10 \mathrm{~Gy} \times 5$ fractions to the PTV were included in the study. These plans were regenerated using BrainLab Monte Carlo (BL_MC), Eclipse AAA (EC_AAA), Eclipse AXB (EC_AXB) and ADAC Pinnacle CCC (AP_CCC) algorithms. The DVH of the PTV was used to calculate dosimetric and radiobiological quality indices, and EQD2 using LQ-L model. The BL_MC algorithm is considered gold standard tool to compare PTV parameters and quality indices to investigate dose calculation discrepancies of abovementioned plans.

The conclusions of this study are BL_PB algorithms overestimates PTV doses than BL_MC calculated doses. The EC_AAA, EC_AXB and AP_CCC algorithms calculate the doses within acceptable limits of radiotherapy dose delivery recommendations.

In the paper by Padannayil, Abdullah, Subha and Sadanadan, authors undertake a study to evaluate the impact of couch translational shifts on DVH and radiobiological parameters [tumour control probability (TCP), equivalent uniform dose (EUD) and normal tissue complication probability (NTCP)] of VMAT plans and to develop a simple and swift method to predict the same online, on a daily basis.

Ten prostate patients treated with VMAT technology were selected for this study. The plans were generated using Eclipse TPS (Version 10; Varian Medical Systems, Palo Alto, CA, USA) and delivered using Clinac ix (Varian Medical Systems) LINAC equipped with a Millennium 120 multileaf collimator. In order to find the effect of systematic translational couch shifts on the DVH and radiobiological parameters, errors were introduced in the clinically accepted base plan with an increment of $1 \mathrm{~mm}$ and up to $5 \mathrm{~mm}$ from the iso-centre in both positive and negative directions of each of the three axis, $x(R-L), y(S-I)$ and $z(A-P)$. The percentages of difference in these parameters $(\triangle \mathrm{D}, \triangle \mathrm{TCP}, \triangle \mathrm{EUD}$ and $\triangle \mathrm{NTCP})$ were analysed between the base plan and the error introduced plans. DVHs of the base plan and the error plans were imported into the MATLAB software (R2013a, The MathWorks, Natick, MA, USA) and an in-house MATLAB code was generated to find the best curve fitted polynomial functions for each point on the DVH, thereby generating predicted DVH for PTV, clinical target volume and OARs. Functions $\mathrm{f}(\mathrm{x}, \mathrm{vj}), \mathrm{f}(\mathrm{y}, \mathrm{vj})$ and $\mathrm{f}(\mathrm{z}, \mathrm{vj})$ were found to represent the variation in the dose when there are couch translation shifts in R-L, S-I and A-P directions, respectively. The validation of this method was done by introducing daily couch shifts and comparing the TPS generated DVHs and radiobiological parameters with MATLAB code predicted parameters.

Authors conclude the variations in the parameters depend upon the direction and magnitude of the shift. The DVH curves generated by the TPS and predicted by the MATLAB showed good correlation.

The technical note in this issue is by Cheung, Sang, Lam, Chan, Wu, Choi, Ho and Law, who present their study to investigate whether significant difference exists on radiation dose delivered to OARs in MVCT verification using three predefined scanning modes, namely fine $(2 \mathrm{~mm})$, normal $(4 \mathrm{~mm})$ and coarse $(6 \mathrm{~mm})$. This 
will provide information for the imaging protocol of tomotherapy for the left breast.

Organ doses were measured using thermoluminescent dosimeters (TLD-100) placed within a female Rando phantom for MVCT imaging. Kruskal-Wallis test was conducted with $p<0.05$ to evaluate the significant difference between the three MVCT scanning modes.

The authors conclude that optimisation of imaging protocols is of paramount importance to keep the radiation exposure 'as low as reasonably achievable'. The recommendation of undergoing daily coarse mode for MVCT verification in breast tomotherapy not only mitigates the radiation exposure to normal tissues, but also trims the scan-acquisition time.
To complete this issue there is a case report on the radiation therapists' compliance to a palliative imaging protocol. Authors Cvetkova, O Donovan, Craig and Mullaney describe a retrospective review of the radiation therapists' compliance to a palliative imaging protocol in a single large institution in one calendar year.

The review showed a non-compliance to protocol for $8 \%$ of treatments. The most frequent protocol deviation was a failure to calculate the mean set-up displacement after $2 / 3$ days of consecutive imaging. Findings are that despite the presence of institutional evidence-based palliative imaging protocol unwanted deviations in practice can occur.

Professor Angela Duxbury 